\title{
Workings OF THE MELTING POT: SOCIAL NETWORKS AND THE EVOLUTION OF POPULATION ATTRIBUTES
}

\author{
JAN K. BRUECKNER \\ OLEG SMIRNOV
}
CESIFO WORKING PAPER No. 1320
CATEGORY 4: LABOUR MARKETS
OCTOBER 2004




\title{
WORKINGS OF THE MELTING Pot: SOCIAL NETWORKS AND THE EVOLUTION OF POPULATION ATTRIBUTES
}

\begin{abstract}
This paper links the two nascent economic literatures on social networks and cultural assimilation by investigating the evolution of population attributes in a simple model where agents are influenced by their acquaintances. The main conclusion of the analysis is that attributes converge to a melting-pot equilibrium, where everyone is identical, provided the social network exhibits a sufficient degree of interconnectedness. When the model is extended to allow an expanding acquaintance set, convergence is guaranteed provided a weaker interconnectedness condition is satisfied, and convergence is rapid. If the intensity of interactions with acquaintances becomes endogenous, convergence (when it occurs) is slowed when agents prefer to interact with people like themselves and hastened when interaction with dissimilar agents is preferred.
\end{abstract}

JEL Code: J00, J10.

Jan K. Brueckner Department of Economics

University of Illinois at Urbana-Champaign

1206 South Sixth St.

Champaign, IL 61820

$$
\text { USA }
$$

jbrueckn@uiuc.edu

\author{
Oleg Smirnov \\ Department of Geography \\ University of Illinois \\ at Urbana-Champaign \\ 607 South Mathews Ave. \\ Urbana, IL 61801 \\ USA \\ osmirnov@uiuc.edu
}




\title{
Workings of the Melting Pot: Social Networks and the Evolution of Population Attributes
}

by

\author{
Jan K. Brueckner and Oleg Smirnov*
}

\section{Introduction}

After early pioneering work in sociology, research on social networks has been a growing focus of economists. Following the important papers of Jackson and Wolinsky (1996) and Bala and Goyal (2000), one branch of the literature analyzes the endogenous formation of network linkages. The goal is to determine the network structure that emerges in equilibrium, while also appraising its efficiency (see Jackson (2004) for a survey). A second branch of the literature, which views the network as exogenously fixed, analyzes the connection between network structure and interactive behavior such as information exchange and learning. The relevant research is surveyed by Goyal (2003). ${ }^{1}$

Another nascent literature, which is tangentially connected to the research on learning in networks, focuses on cultural assimilation in heterogeneous societies. The goal is to investigate the evolution of population attributes in models where parents must invest resources in order to transmit a cultural identity to their children, overcoming external socialization influences. Using such an approach, Bisin and Verdier (2001) derive conditions under which population heterogeneity is a long-run equilibrium, with assimilation not occurring. By contrast, Kónya (2002) shows in a related model that a minority group will be assimilated (remain distinct) when its initial population share is small (large). ${ }^{2}$

Borrowing elements from both these literatures, the goal of the present paper is to analyze the effect of social networks on the evolution of population attributes, which occurs as agents interact via the network. As in the second branch of the network literature, the pattern of network linkages is exogenously specified, with individuals who are directly linked called "acquaintances." Exogeneity of the network is matched by an equally simple assumption regarding the law of motion of population attributes, which does not involve in any economic decisions 
by individual agents. In particular, agent $i$ 's attributes, which are summarized by a scalar variable $\theta_{i}$, are influenced by the attributes of his acquaintances, as follows: $i$ 's attributes at time $t+1$ equal the average attributes among his acquaintances at time $t$. Under one assumption, this averaging includes the agent himself, but alternatively, the agent's attributes at time $t+1$ may not depend on their previous value. This law of motion embodies the natural view that the social, political and cultural traits of individual agents tend to evolve toward the traits of the people they know.

The goal of the analysis is to investigate the long-run evolution of population attributes under this simple law of motion and to show how it depends on the structure of the social network. The analysis demonstrates that, if the social network is sufficiently interconnected, in several senses to be made precise below, then population attributes converge to a "meltingpot" equilibrium. In such an equilibrium, the attributes of all agents are identical and equal to the mean of the population's initial attribute values. When the interconnection requirement is not satisfied (a result of simultaneous violation of two sufficient conditions), then a melting-pot equilibrium may not be reached. Instead, population attributes may vary across agents, while cycling over time. These results, which are derived by drawing on the theory of irreducible matrices, demonstrate the workings of the melting pot in the simplest possible framework.

Although the basic model assumes that an agent's set of acquaintances remains constant, the first extension of the model allows the acquaintance set to grow over time. In particular, an individual's acquaintance set at time $t+1$ is assumed to consist of his time- $t$ acquaintances along with the acquaintances of these acquaintances at time $t$. Thus, in each period, the acquaintances of $i$ 's last-period acquaintances are added to his acquaintance set. The analysis shows that, when acquaintance sets expand according to this rule, convergence to a meltingpot equilibrium occurs as long as the social network satisfies a minimal interconnectedness condition. In addition, a numerical example shows that convergence is rapid compared to the basic model.

While the basic model is devoid of any economic behavior, with the network and the law of motion lacking any endogenous elements, such elements are introduced in the second extension of the model. In that extension, the intensity of interaction is a choice variable 
whose determination depends on agents' attitudes toward dissimilar individuals. One possible assumption is that people interact more intensively with agents like themselves. ${ }^{3}$ Alternatively, diversity could be valued, with the strength of interaction rising with the dissimilarity of acquaintances. The law of motion is then modified so that an agent's attributes at time $t+1$ are an interaction-intensity weighted average of the attributes of his acquaintances at time $t$. Although general results are not available for this modified model, numerical examples show that, when melting-pot convergence occurs, it happens more slowly than in the basic model under the first interaction assumption and faster under the second.

It is important to note that the analysis in the paper does not focus on any of the economic consequences of melting-pot convergence, which might be considered in a more comprehensive model. The nature of such consequences could be inferred from the survey paper of Alesina and La Ferrara (2004), which reviews the economic literature on the effects of racial diversity. The empirical part of that literature shows that one impact of racial diversity is to reduce economic

growth, both at the country and subnational levels. If such an effect also applies to a more general set of population attributes like that considered in this paper, then, by homogenizing the population, melting-pot convergence produces economic benefits. Such benefits could be captured in a more extensive model.

The plan of the paper is as follows. Section 2 analyzes the basic model, while section 3 develops the extensions. Section 4 offers conclusions.

\section{Basic Analysis}

\subsection{The acquaintance matrix}

The population consists of $m$ agents, and the attributes of each individual are summarized by a scalar variable, as explained above. The characteristics of agent $i$ at time $t$ are denoted $\theta_{i}^{t}, i=1, \ldots, m$.

Each agent is acquainted with other agents in the population, and the pattern of acquaintances is captured by the variables $n_{i j}$, for $i \neq j$. If agent $i$ is acquainted with agent $j$, then $n_{i j}=1$, while if the agents are unacquainted, then $n_{i j}=0$. Given the symmetry of acquaintances, it follows that $n_{i j}=n_{j i}$. The variables $n_{i i}$ have a different status since they refer 
to an agent's relationship to himself. Accordingly, the cases where $n_{i i}=1$ and $n_{i i}=0$ are both considered, with the interpretation of the latter case provided below. The number of acquaintances for agent $i$ equals $q_{i}=\sum_{j=1}^{m} n_{i j}$, where $i$ himself is counted as an acquaintance if $n_{i i}=1$. It is assumed that $q_{i}>0$ for all $i$. Finally, the acquaintance pattern is summarized by the symmetric matrix $N$, which has representative element $n_{i j}$. In the following discussion, $N$ will be referred to as the raw acquaintance matrix.

A key assumption in the analysis is that the matrix $N$ is irreducible. This assumption means that any two agents are linked indirectly by a sequence of acquaintances through other people, even though the agents may not be acquainted themselves. Formally, irreducibility means that, for all $(i, j), N_{i j}^{(k)}>0$ holds for some $k>0$, where this expression refers to element $(i, j)$ of the $k$ th power of the matrix $N$ (the resulting matrix is $N^{k}$ ). As seen from discussion below, $N_{i j}^{(k)}$ equals the number of paths of length $k$ connecting agents $i$ and $j$ via other agents. Therefore, the irreducibility requirement means that each pair of agents is connected by a path of some length.

To see the relationship between connecting paths and the powers of $N$, consider the following matrix $N$ and its square:

$$
N=\left(\begin{array}{lllll}
0 & 1 & 0 & 0 & 1 \\
1 & 0 & 1 & 0 & 0 \\
0 & 1 & 0 & 1 & 0 \\
0 & 0 & 1 & 0 & 1 \\
1 & 0 & 0 & 1 & 0
\end{array}\right), \quad N^{2}=\left(\begin{array}{lllll}
2 & 0 & 1 & 1 & 0 \\
0 & 2 & 0 & 1 & 1 \\
1 & 0 & 2 & 0 & 1 \\
1 & 1 & 0 & 2 & 0 \\
0 & 1 & 1 & 0 & 2
\end{array}\right)
$$

The acquaintance pattern in $N$ would be generated if the agents were arrayed on circle, being acquainted with their immediate neighbors (note that agents 1 and 5 are adjacent and that all the $n_{i i}$ 's are set to zero for illustrative purposes). Since all agents are connected by some path around the circle, the given $N$ is irreducible.

Looking at $N^{2}$, the fact that element $(1,3)$ equals 1 indicates that there is a single path of length 2 between agents 1 and 3 . This path is of the form $1-2-3$, with agent 1 connected to 3 via his acquaintance with 2 and 2's acquaintance with 3 . Similarly, the single path $1-5-4$ exists between agents 1 and 4, and the two paths $1-2-1$ and $1-5-1$ exist between agent 1 and himself. However, the remaining zeros in the first row of $N^{2}$ indicate that there 
are no paths of length 2 between agents 1 and 2 or between agents 1 and 5 . Raising $N$ to an additional power, yielding $N^{3}$, would eliminate these zeros, indicating the existence of length-3 paths between these pairs of agents. In this fashion, the irreducibility assumption says that element $(i, j)$ of $N^{k}$ cannot be zero for all possible values of $k .^{4}$

As an example of a raw acquaintance matrix that is not irreducible (i.e., reducible), consider the case where $N$ is a block diagonal matrix. In this case, the population is divided into separate groups (indicated by the blocks), between which linkages are absent. The zero offdiagonal blocks remain zero as the matrix is raised to higher powers, indicating failure of the requirement of irreducibility.

\subsection{The law of motion for population attributes}

As explained in the introduction, the evolution of population attributes is determined by a simple rule in the basic model. In particular, agent $i$ 's attributes at time $t+1$ equal the average attributes at time $t$ among his acquaintances (which may include himself). The law of motion for attributes can thus be written

$$
\theta_{i}^{t+1}=\sum_{j=1}^{m}\left(\frac{n_{i j}}{q_{i}}\right) \theta_{j}^{t}
$$

Note that when $n_{i i}=1$, this rule says that agent $i$ 's attributes at time $t+1$ are a blend of his own attributes and those of his other acquaintances at time $t$. By contrast, when $n_{i i}=0$, agent $i$ 's time- $t$ attributes play no role in determining his attributes at time $t+1$, which depend only on the attributes of his (other) acquaintances. In the ensuing discussion, an agent will be called "self-referential" if $n_{i i}=1$, indicating that his future attributes depend on their current value.

To rewrite the law of motion from (2) in more compact form, let $A$ denote the rownormalized acquaintance matrix (shortened to "acquaintance matrix" in the ensuing discussion). This matrix is generated from the raw acquaintance matrix by dividing each element of the $i$ th row of $N$ by the row sum, which equals $q_{i}$, for $i=1, \ldots, m$. A representative element of $A$ is then $n_{i j} / q_{i} \equiv a_{i j}$. Thus, if the first row of $N$ contains four 1's and $m-4$ zeros, then first row of $A$ has a $1 / 4$ in each of the spots where $N$ has a 1 , and zeros elsewhere. It is easy 
to see that if the raw acquaintance matrix is irreducible, as assumed, the same property holds for the matrix $A$.

Recognizing that $(2)$ can be written as $\theta_{i}^{t+1}=\sum_{j=1}^{m} a_{i j} \theta_{j}^{t}$, the law of motion can be rewritten in matrix form as

$$
\theta^{t+1}=A \theta^{t}
$$

where $\theta^{t+1}$ is the column vector $\left(\theta_{1}^{t+1} \theta_{2}^{t+1} \ldots \theta_{m}^{t+1}\right)^{\prime}$ and $\theta^{t}$ is defined analogously.

Suppose that the evolutionary process for attributes starts at time zero. Then with successive substitutions, (3) becomes

$$
\theta^{t}=A^{t} \theta^{0}
$$

where $\theta^{0}=\left(\begin{array}{llll}\theta_{1}^{0} & \theta_{2}^{0} & \cdots & \theta_{m}^{0}\end{array}\right)^{\prime}$ is the population attribute vector at time zero and $A^{t}$ is the $t$ th power of the acquaintance matrix. If the evolutionary process converges to a limit, the limiting attribute vector is given by

$$
\lim _{t \rightarrow \infty} \theta^{t}=\theta^{*} \equiv A^{*} \theta^{0}
$$

where

$$
A^{*}=\lim _{t \rightarrow \infty} A^{t}
$$

which must exist for the process to converge.

\subsection{Analysis of convergence}

To analyze convergence, it is useful to initially focus on the case where each individual has the same number of acquaintances, with $q_{i}$ equal to a constant $q$ for all $i$. In this case, the symmetry of $N$ is preserved under normalization, with $A$ equal to $N$ divided by $q$. Then, to investigate the limit of $A^{t}$, it is useful to write the acquaintance matrix in diagonalized form. To do so, let $\lambda_{i}, i=1, \ldots, m$, denote the eigenvalues of $A$, which are assumed to be distinct (given symmetry of $N$, these eigenvalues are real). Then, in standard fashion, $A$ can be written

as $S D S^{-1}$, where $S$ is a matrix whose columns are the eigenvectors of $A$, and where $D$ is a matrix whose diagonal elements equal $\lambda_{i}, i=1, \ldots, m$, and whose off-diagonal elements equal 
zero. With this representation of the acquaintance matrix, $A^{t}$ can be written as ${ }^{5}$

$$
A^{t}=\left(S D S^{-1}\right)\left(S D S^{-1}\right)\left(S D S^{-1}\right) \cdots\left(S D S^{-1}\right)=S D^{t} S^{-1}
$$

Symmetry of $A$ means that, $S^{-1}$, the inverse of the matrix of eigenvectors of $A$, is simply equal to the transpose of that matrix, $S^{\prime}$. As a result, $(7)$ can be written

$$
A^{t}=S D^{t} S^{\prime}=\left(\begin{array}{llll}
\lambda_{1}^{t} S_{1} & \lambda_{2}^{t} S_{2} & \cdots & \lambda_{m}^{t} S_{m}
\end{array}\right)\left(\begin{array}{c}
S_{1}^{\prime} \\
S_{2}^{\prime} \\
\cdot \\
\cdot \\
\cdot \\
S_{m}^{\prime}
\end{array}\right)
$$

where $S_{i}$ is the $i$ th eigenvector (the $i$ th column of $\left.S\right){ }^{6}$

To make use of (8), key facts about irreducible matrices can be invoked. First, the PerronFrobenius Theorem and its corollaries state that the largest eigenvalue of an irreducible matrix lies between the maximum and minimum row sums of the matrix. Since row-normalization makes the row sums of $A$ all equal to unity, it follows that $A$ 's largest eigenvalue equals 1. Second, no eigenvalue of $A$ exceeds 1 in absolute value. Third, $A$ may have a second eigenvalue equal to -1 , but all other eigenvalues are less than one in absolute value (see Seneta (1973), ch. 1). When $A$ has an eigenvalue equal to -1 , it is known as a cyclic matrix, for reasons that will become clear below. When a -1 eigenvalue does not exist, $A$ is a noncyclic matrix.

Consider the case where $A$ is noncyclic, and let the unitary eigenvalue correspond to $\lambda_{1}$. Then, since the remaining eigenvalues are all less than one in absolute value, $\lambda_{i}^{t}$ converges to zero for $i=2, \ldots, m$. As a result, the last $m-1$ columns of the first matrix in (8) themselves converge to zero, so that the matrix converges to $\left(S_{1} 00 \cdots 0\right)$. The matrix product in $(8)$ then converges to $S_{1} S_{1}^{\prime}$, an $m \times m$ matrix.

To evaluate this matrix, the eigenvector corresponding to the unitary eigenvalue must be found. This is a simple matter, however, since it is easily seen that the equation $A x=1 x$ is satisfied by the unit vector $x=\left(\begin{array}{llll}1 & 1 & \cdots\end{array}\right)^{\prime}$, given that $A$ is row normalized. Dividing this 
vector by $\sqrt{m}$ to give it unitary length, $S_{1}$ can be written $\left(\frac{1}{\sqrt{m}} \frac{1}{\sqrt{m}} \cdots \frac{1}{\sqrt{m}}\right)^{\prime}$. As a result,

$$
A^{*}=\lim _{t \rightarrow \infty} A^{t}=S_{1} S_{1}^{\prime}=\left(\begin{array}{cccc}
1 / m & 1 / m & \cdots & 1 / m \\
1 / m & 1 / m & \cdots & 1 / m \\
\cdot & \cdot & & \cdot \\
1 / m & 1 / m & \cdots & 1 / m
\end{array}\right)
$$

Using (5), it follows then that

$$
\theta^{*}=A^{*} \theta^{0}=\left(\begin{array}{c}
\bar{\theta}^{0} \\
\bar{\theta}^{0} \\
\cdot \\
\cdot \\
\bar{\theta}^{0}
\end{array}\right)
$$

where

$$
\bar{\theta}^{0}=\frac{1}{m} \sum_{i=1}^{m} \theta_{i}^{0},
$$

the mean attributes of the population at time zero.

Thus, when $A$ is noncyclic, the evolutionary process for population attributes converges to a "melting-pot" equilibrium. In this equilibrium, attributes are uniform across the population, with each agent having attributes equal to the average initial value.

To understand how this convergence occurs, consider three agents, $i, j$ and $k$, and suppose that $i$ and $k$ are unacquainted but that $i-j$ and $j-k$ acquaintance links exist. Since $j$ 's attributes therefore depend on $k$ 's while at the same time influencing $i$ 's attributes, it follows that $i$ partly adopts $k$ 's attributes as the population evolves, even though the two agents are not acquainted. When $A$ is noncyclic, this process leads to a melting-pot equilibrium.

To analyze the cyclic case, let $\lambda_{2}$ denote $A^{\prime}$ 's -1 eigenvalue. Because $\lambda_{i}^{t}$ still converges to zero for $i \geq 3$, repetition of the previous argument shows that $\theta^{t}$ approaches

$$
\left(\begin{array}{c}
\bar{\theta}^{0} \\
\bar{\theta}^{0} \\
\cdot \\
\cdot \\
\dot{\theta}^{0}
\end{array}\right)+(-1)^{t} S_{2} S_{2}^{\prime} \theta^{0}
$$


as $t$ becomes large, where $S_{2}$ is the eigenvector corresponding to $\lambda_{2}$. Thus, attributes for any given agent will oscillate around the value $\bar{\theta}^{0}$, with the vector $S_{2} S_{2}^{\prime} \theta^{0}$ successively added and subtracted from the vector $\left(\bar{\theta}^{0} \bar{\theta}^{0} \ldots \bar{\theta}^{0}\right)$ as time progresses. Since it can be shown that the mean attribute level remains constant at $\bar{\theta}^{0}$ during the evolutionary process when $A$ is symmetric, the elements of $S_{2} S_{2}^{\prime} \theta^{0}$ must differ in sign. ${ }^{7}$ As a result, the oscillation will be unsynchronized for different individuals, with attribute values stepping up for some agents as they step down for the others.

Thus, rather than converging to the stable, uniform value of the melting-pot equilibrium, population attributes in this cyclic case never settle down, cycling over time while showing nonuniformity across agents. Summarizing yields

Proposition 1. Suppose that each agent has the same number of acquaintances and that the acquaintance matrix $A$ is irreducible. If $A$ is noncyclic, then population attributes converge to a melting-pot equilibrium, where attributes for each agent are equal to the mean initial value in the population. If $A$ is instead cyclic, a melting-pot equilibrium is not achieved, with population characteristics cycling over time and exhibiting nonuniformity across agents.

Now consider the case where the number of acquaintances differs across agents. In this case $A$, does not share the symmetry of $N$, which means that the simplification that leads from (7) to (8) is unavailable. A conclusion similar to Proposition 1 can be established, however, by appealing to the general result of Seneta (1973). His Theorem 1.2 shows that, when $A$ is noncyclic, $\theta^{t}$ converges to $S_{1 r} S_{1 l}^{\prime} \theta^{0}$, where $S_{1 r}$ and $S_{1 l}$ are right and left eigenvectors corresponding to the unitary eigenvalue. $S_{1 r}$ is the same as the eigenvector $\left(\frac{1}{\sqrt{m}} \frac{1}{\sqrt{m}} \cdots \frac{1}{\sqrt{m}}\right)^{\prime}$ from above, while the left eigenvalue satisfies $S_{1 l}^{\prime} A=S_{1 l}^{\prime}$. Note that while the positive elements in a row of $A$ are the same (equal to $1 / q_{i}$ for row $i$ ), nonuniformity of the $q_{i}$ 's means that the positive elements in a given column of $A$ will differ. As a result, the left and right eigenvectors need not be equal. Observe, however, that in the previous case where $A$ is symmetric, $S_{1 r}=$ $S_{1 l} \equiv S_{1}$ holds and Seneta's Theorem 1.2 can be used directly to reach (9) and (10). 
Substituting for $S_{1 r}$, the expression $S_{1 r} S_{1 l}^{\prime} \theta^{0}$ can be written as

$$
\left(\begin{array}{c}
1 / \sqrt{m} \\
1 / \sqrt{m} \\
\cdot \\
\cdot \\
\cdot \\
1 / \sqrt{m}
\end{array}\right) S_{1 l}^{\prime} \theta^{0}=\left(\begin{array}{cccc}
S_{1 l, 1} / \sqrt{m} & S_{1 l, 2} / \sqrt{m} & \ldots & S_{1 l, m} / \sqrt{m} \\
S_{1 l, 1} / \sqrt{m} & S_{1 l, 2} / \sqrt{m} & \ldots & S_{1 l, m} / \sqrt{m} \\
\cdot & \cdot & & \cdot \\
S_{1 l, 1} / \sqrt{m} & S_{1 l, 2} / \sqrt{m} & \ldots & S_{1 l, m} / \sqrt{m}
\end{array}\right) \theta^{0}=\left(\begin{array}{c}
\hat{\theta}_{0} \\
\hat{\theta}_{0} \\
\cdot \\
\cdot \\
\cdot \\
\hat{\theta}_{0}
\end{array}\right)
$$

where $S_{1 l, i}$ is the $i$ th element of $S_{1 l}$. In (13), $\hat{\theta}_{0}$ is some weighted sum of the $\theta_{i}^{0}$ values, which does not necessarily equal the mean value. Summarizing yields

Proposition 2. When the number of acquaintances differs across agents and $A$ is irreducible and noncyclic, then population attributes again converge to a melting-pot equilibrium, with attributes uniform across the population but not necessarily equal to the initial mean. When $A$ is cyclic, the melting-pot equilibrium is not achieved.

Note that in the latter case, the time path of attributes is not given explicitly (as in (12)), but it is clear from (7) that attributes will cycle over time and not necessarily exhibit uniformity across the population.

\subsection{The social network and convergence}

Whether or not a melting-pot equilibrium is achieved depends on the eigenvalues of $A$, and their magnitudes in turn depend on the characteristics of the social network. Insight into this connection can be gained through the notion of the period of an irreducible matrix, denoted $d$. If the acquaintance matrix $A$ is noncyclic, then it has $d=1$. A cyclic matrix has $d>1$, but for an irreducible, row-normalized matrix like $A, d$ equals $2 .^{8}$

$A$ 's period depends on the periods of its indices, $i=1,2, \ldots, m$. The period of index $i$, denoted $d_{i}$, is the greatest common divisor of those $k$ values for which $A_{i i}^{(k)}>0$. Recall that $A_{i i}^{(k)}$ denotes the $i$ th diagonal element of the $k$ th power of matrix $A$. For example, if $A_{i i}^{(k)}>0$ holds for all even $k$ 's starting with $6(k=6,8,10, \ldots)$, then $d_{i}=2$. Similarly, if $A_{i i}^{(k)}>0$ holds for all $k \geq 2$, then $d_{i}=1$. A key property of any irreducible matrix is that all its indices have the same period, which then gives the period $d$ of the matrix.

It can be difficult to determine the period of a particular acquaintance matrix without actually computing its powers. However, in some general cases, it is easy to verify that a 
matrix has $d=1$, establishing noncyclicality. Two such cases, where a melting-pot equilibrium is guaranteed to emerge, are considered in the following discussion. The next section then presents numerical examples illustrating these cases as well as a cyclical case.

To explore the first noncyclical example, suppose that some agent $j$ is self-referential. Recall that this property means that $n_{j j}=1$, so that, under the law of motion in (2), agent $j$ 's time- $(t+1)$ attributes depend partly on his attributes at time $t$. Since $n_{j j}=1$, it follows that $a_{j j}$, the corresponding diagonal element of $A$, is positive. As result, the inner product of the $j$ th row of $A$ and the (identical) $j$ th column, which gives $A_{j j}^{(2)}$, contains the term $a_{j j}^{2}>0$. Since the nonnegativity of all the elements of $A$ means that any remaining terms in this inner product are nonnegative, it follows that $A_{j j}^{(2)}>0$. But with this diagonal element positive, the inner product of the $j$ th row of $A$ and the $j$ th column of $A^{2}$ again produces a positive value, which equals $A_{j j}^{(3)}>0$. Continuing this progression, it is clear $A_{j j}^{(k)}>0$ holds for all positive $k$, implying that $d_{j}=1$. This conclusion in turn implies that $d=1$ and hence that $A$ is noncyclic, yielding

Proposition 3. If at least one agent is self-referential, then $A$ is noncyclic and the evolution of population attributes leads to a melting-pot equilibrium.

This conclusion is intuitively sensible given that pure mimicking behavior, where agents just look to others as attributes evolve, would appear to raise the possibility of an unstable evolutionary process. Remarkably, though, it takes just one agent to be self-referential, and thus "grounded" by his own attributes, to get convergence to a melting-pot equililbrium. In the next section, a numerical example shows that universal mimickry, where no agent is selfreferential, can generate the cyclical case.

To develop the second noncyclical example, suppose that no agent is self-referential, so that $A$ has zero diagonal elements. But suppose that two agents, say 1 and 2, are aquainted with each other and that both are acquainted with a common third person, agent 3 . Then $A$ 
has the following form:

$$
\left(\begin{array}{cccc}
0 & 1 / q_{1} & 1 / q_{1} & \cdots \\
1 / q_{2} & 0 & 1 / q_{2} & \cdots \\
1 / q_{3} & 1 / q_{3} & 0 & \cdots \\
\cdot & \cdot & \cdot & \\
\cdot & \cdot & \cdot & \\
\cdot & \cdot & \cdot &
\end{array}\right)
$$

(note that no assumptions are made about the structure of $A$ outside of its upper corner). Using (14), the first column of $A^{2}$ is equal to

$$
\left(\begin{array}{c}
\frac{1}{q_{1} q_{2}}+\frac{1}{q_{1} q_{3}}+e \\
\frac{1}{q_{2} q_{3}}+f \\
\frac{1}{q_{2}^{2}}+g \\
\cdot \\
\cdot
\end{array}\right)
$$

where $e, f$ and $g$ are nonnegative values. Since the first three elements of (15) are positive, while the first three rows of $A$ each have two of their first three elements positive, it follows that the first column of $A^{3}$ has the form of (15), with the first three elements again positive. Continuing this progression, it follows that the first three elements of $A^{k}$ 's first column are always positive for $k \geq 2$. As a result, the diagonal element $A_{11}^{(k)}$ is positive for $k \geq 2$, which implies $d_{1}=1$ and hence $d=1$. Since agents 1,2 and 3 were chosen arbitrarily, this argument yields

Proposition 4. If any two agents are acquainted with a third common agent, then $A$ is noncyclic and the evolution of population attributes leads to a melting-pot equilibrium.

The existence of common acquaintances clearly makes the population more integrated, facilitating the blending of attributes across agents. As in the case of Proposition 3, however, only one case of such integration is needed to ensure convergence to a melting-pot equilibrium.

The implication of Propositions 3 and 4 is that convergence to a melting-pot equilibrium in the model can be expected under a wide variety of social-network structures. The network needs just one self-referential agent or two individuals with a common acquaintance to rule out 
the nonconvergence outcome. The weakness of these requirements suggests that convergence is the natural outcome under the model.

\subsection{Numerical examples}

This section presents numerical examples to illustrate some of the points developed above. Consider a setting with 10 agents, each of whom is self-referential and has two additional acquaintances. Let the raw acquaintance matrix for this case be given by

$$
N=\left(\begin{array}{llllllllll}
1 & 1 & 0 & 0 & 0 & 0 & 0 & 0 & 0 & 1 \\
1 & 1 & 1 & 0 & 0 & 0 & 0 & 0 & 0 & 0 \\
0 & 1 & 1 & 1 & 0 & 0 & 0 & 0 & 0 & 0 \\
0 & 0 & 1 & 1 & 1 & 0 & 0 & 0 & 0 & 0 \\
0 & 0 & 0 & 1 & 1 & 1 & 0 & 0 & 0 & 0 \\
0 & 0 & 0 & 0 & 1 & 1 & 1 & 0 & 0 & 0 \\
0 & 0 & 0 & 0 & 0 & 1 & 1 & 1 & 0 & 0 \\
0 & 0 & 0 & 0 & 0 & 0 & 1 & 1 & 1 & 0 \\
0 & 0 & 0 & 0 & 0 & 0 & 0 & 1 & 1 & 1 \\
1 & 0 & 0 & 0 & 0 & 0 & 0 & 0 & 1 & 1
\end{array}\right)
$$

Recall that this acquaintance pattern would be generated if the agents were arrayed on circle, being acquainted with their immediate neighbors. Finally, suppose that an agent's initial

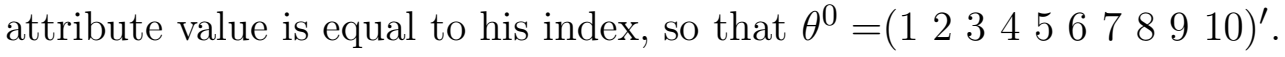

The evolutionary process for this base case is shown in Table 1 . Since each agent has 3 acquaintances, while the existence of self-referential agents ensures converge to the melting-pot equilibrium, Proposition 1 applies. As can be seen, attributes converge to 5.50, the mean of the initial values, by period $100 .^{9}$

Table 2 shows the effect of making each agent non-self-referential, which means that all the diagonal elements of $N$ in (16) are set equal to zero. Convergence is not guaranteed in this case, and as can be seen from the table, convergence indeed does not occur. Once $t$ reachs 64, the attributes of each agent cycle back and forth between 5.00 and 6.00 as $t$ increases further. At each point in time, attributes are non-uniform across the population, indicating the absence of a melting-pot outcome. ${ }^{10}$

To illustrate Proposition 3, Table 3 shows the effect of making agent 1 self-referential, which means setting $N$ 's first diagonal element to 1 while leaving the others at zero. Consistent with 
the predictions of the Proposition 3, Table 3 shows convergence to a melting-pot equilibrium by $t=125 .^{11}$ However, since the number of acquaintances is now non-uniform (with agent 1 having 3 and the other agents having 2 acquaintances), convergence is to an attribute value of 5.28 , which less than the mean value of 5.50 .

Finally, Table 4 illustrates Proposition 4 by setting all $N$ 's diagonal elements to zero but assuming that agent 1 is acquainted with agent 3 as well as agents 2 and 10, so that both agents 1 and 2 have 3 as a common acquaintance. This change means setting the $(1,3)$ and $(3,1)$ elements of $N$ to 1 , as in the case illustrated in (14) (recall, however, that (14) shows $A$, not $N$ ). As predicted by Proposition 4, Table 4 shows convergence to a melting-pot equilibrium by $t=100$, but given non-uniform aquaintance numbers, convergence is again to an attribute value less than the initial mean. ${ }^{12}$

\subsection{Attribute evolution when $N$ is reducible}

It is instructive to briefly consider case where $N$ is block diagonal and thus reducible. Recall that in this case, linkages between the groups corresponding to the blocks are absent,

so that $N_{i j}^{(k)}=0$ for all $k$ when $(i, j)$ represents an entry in one of the zero off-diagonal blocks. In this case, it is easy to see that, as long as the submatrices corresponding to the individual diagonal blocks are themselves irreducible and noncyclic, convergence to a "localized" meltingpot equilibrium occurs within each block. In other words, for each agent within a given block, attributes converge to the initial block average. For example, keeping the structure of the numerical examples above, suppose that $N$ has two blocks corresponding to $i=1,2,3,4$ and $i=5,6,7,8,9,10$. Then attributes for agents in the first and second blocks converge to $\theta=2.5$ and $\theta=7.5$ respectively, provided that the corresponding block submatrices are irreducible and noncyclic.

It is interesting to note, however, that creation of just one link between the groups represented by the diagonal blocks makes $N$ irreducible, leading to global melting-pot convergence. For example, if agents 4 and 5 are acquainted, which converts the $(4,5)$ and $(5,4)$ elements of the two zero blocks from 0's to 1's, then convergence to a melting-pot equilbrium occurs. ${ }^{13}$ 
Table 1

Attribute Evolution in the Base Case

\begin{tabular}{rrrrrrrrrrr}
\hline \hline$t$ & $\theta_{1}$ & $\theta_{2}$ & $\theta_{3}$ & $\theta_{4}$ & $\theta_{5}$ & $\theta_{6}$ & $\theta_{7}$ & $\theta_{8}$ & $\theta_{9}$ & $\theta_{10}$ \\
\hline 0 & 1.00 & 2.00 & 3.00 & 4.00 & 5.00 & 6.00 & 7.00 & 8.00 & 9.00 & 10.00 \\
3 & 4.70 & 3.48 & 3.37 & 4.00 & 5.00 & 6.00 & 7.00 & 7.62 & 7.51 & 6.29 \\
10 & 5.24 & 4.82 & 4.67 & 4.83 & 5.24 & 5.75 & 6.16 & 6.32 & 6.17 & 5.75 \\
30 & 5.48 & 5.45 & 5.44 & 5.45 & 5.48 & 5.51 & 5.54 & 5.55 & 5.54 & 5.51 \\
64 & 5.49 & 5.49 & 5.49 & 5.49 & 5.49 & 5.50 & 5.50 & 5.50 & 5.50 & 5.50 \\
65 & 5.49 & 5.49 & 5.49 & 5.49 & 5.49 & 5.50 & 5.50 & 5.50 & 5.50 & 5.50 \\
100 & 5.50 & 5.50 & 5.50 & 5.50 & 5.50 & 5.50 & 5.50 & 5.50 & 5.50 & 5.50 \\
125 & 5.50 & 5.50 & 5.50 & 5.50 & 5.50 & 5.50 & 5.50 & 5.50 & 5.50 & 5.50 \\
\hline
\end{tabular}

Table 2

Attribute Evolution When No Agents are Self-Referential

\begin{tabular}{rrrrrrrrrrr}
\hline \hline$t$ & $\theta_{1}$ & $\theta_{2}$ & $\theta_{3}$ & $\theta_{4}$ & $\theta_{5}$ & $\theta_{6}$ & $\theta_{7}$ & $\theta_{8}$ & $\theta_{9}$ & $\theta_{10}$ \\
\hline 0 & 1.00 & 2.00 & 3.00 & 4.00 & 5.00 & 6.00 & 7.00 & 8.00 & 9.00 & 10.00 \\
3 & 6.00 & 3.25 & 4.25 & 4.00 & 5.00 & 6.00 & 7.00 & 6.75 & 7.75 & 5.00 \\
10 & 4.75 & 5.75 & 4.61 & 5.61 & 5.00 & 6.00 & 5.38 & 6.38 & 5.24 & 6.24 \\
30 & 4.99 & 5.99 & 4.99 & 5.99 & 5.00 & 6.00 & 5.00 & 6.00 & 5.00 & 6.00 \\
64 & 5.00 & 6.00 & 5.00 & 6.00 & 5.00 & 6.00 & 5.00 & 6.00 & 5.00 & 6.00 \\
65 & 6.00 & 5.00 & 6.00 & 5.00 & 6.00 & 5.00 & 6.00 & 5.00 & 6.00 & 5.00 \\
100 & 5.00 & 6.00 & 5.00 & 6.00 & 5.00 & 6.00 & 5.00 & 6.00 & 5.00 & 6.00 \\
125 & 6.00 & 5.00 & 6.00 & 5.00 & 6.00 & 5.00 & 6.00 & 5.00 & 6.00 & 5.00 \\
\hline
\end{tabular}


Table 3

Attribute Evolution When Only Agent 1 Is Self-Referential

\begin{tabular}{rrrrrrrrrrr}
\hline \hline$t$ & $\theta_{1}$ & $\theta_{2}$ & $\theta_{3}$ & $\theta_{4}$ & $\theta_{5}$ & $\theta_{6}$ & $\theta_{7}$ & $\theta_{8}$ & $\theta_{9}$ & $\theta_{10}$ \\
\hline 0 & 1.00 & 2.00 & 3.00 & 4.00 & 5.00 & 6.00 & 7.00 & 8.00 & 9.00 & 10.00 \\
3 & 4.70 & 3.38 & 3.83 & 4.00 & 5.00 & 6.00 & 7.00 & 6.75 & 7.33 & 5.13 \\
10 & 5.00 & 5.02 & 4.74 & 5.17 & 5.06 & 5.69 & 5.44 & 5.94 & 5.37 & 5.50 \\
30 & 5.25 & 5.33 & 5.20 & 5.37 & 5.17 & 5.40 & 5.18 & 5.39 & 5.20 & 5.34 \\
64 & 5.27 & 5.30 & 5.26 & 5.31 & 5.25 & 5.32 & 5.25 & 5.31 & 5.26 & 5.30 \\
65 & 5.29 & 5.26 & 5.30 & 5.25 & 5.31 & 5.25 & 5.31 & 5.25 & 5.30 & 5.26 \\
100 & 5.28 & 5.28 & 5.29 & 5.27 & 5.29 & 5.27 & 5.29 & 5.27 & 5.29 & 5.28 \\
125 & 5.28 & 5.28 & 5.28 & 5.28 & 5.28 & 5.28 & 5.28 & 5.28 & 5.28 & 5.28 \\
\hline
\end{tabular}

Table 4

Attribute Evolution When Agents 1 and 2 Are Both Acquainted with Agent 3

\begin{tabular}{rrrrrrrrrrr}
\hline \hline$t$ & $\theta_{1}$ & $\theta_{2}$ & $\theta_{3}$ & $\theta_{4}$ & $\theta_{5}$ & $\theta_{6}$ & $\theta_{7}$ & $\theta_{8}$ & $\theta_{9}$ & $\theta_{10}$ \\
\hline 0 & 1.00 & 2.00 & 3.00 & 4.00 & 5.00 & 6.00 & 7.00 & 8.00 & 9.00 & 10.00 \\
3 & 4.77 & 3.38 & 3.48 & 4.33 & 4.83 & 6.00 & 7.00 & 6.75 & 7.50 & 4.80 \\
10 & 4.75 & 4.82 & 4.86 & 4.96 & 5.19 & 5.60 & 5.44 & 5.91 & 5.19 & 5.42 \\
30 & 5.16 & 5.18 & 5.16 & 5.20 & 5.14 & 5.24 & 5.13 & 5.25 & 5.13 & 5.21 \\
64 & 5.18 & 5.18 & 5.18 & 5.18 & 5.17 & 5.18 & 5.17 & 5.18 & 5.17 & 5.18 \\
65 & 5.18 & 5.18 & 5.18 & 5.17 & 5.18 & 5.17 & 5.18 & 5.17 & 5.18 & 5.17 \\
100 & 5.18 & 5.18 & 5.18 & 5.18 & 5.18 & 5.18 & 5.18 & 5.18 & 5.18 & 5.18 \\
125 & 5.18 & 5.18 & 5.18 & 5.18 & 5.18 & 5.18 & 5.18 & 5.18 & 5.18 & 5.18 \\
\hline
\end{tabular}




\section{Extensions}

This section considers two extensions of the model. Under, the first extension, an agent's set of acquaintances grows over time rather than being fixed. Indirect linkages produce new acquaintances in each period, with agents becoming acquainted with the acquaintances of their acquaintances. The second extension allows individuals to choose the intensity of interaction with their acquaintances. The law of motion in (2) is then amended so that an agent's attributes at time $t+1$ are equal to an intensity-weighted average of his acquaintances' attributes at time $t$.

\subsection{The effect of an expanding acquaintance set}

Suppose that an agent's set of acquaintances expands over time through indirect linkages. To formalize this expansion, let the set of agent $i$ 's acquaintances at $t=0$ be denoted by $\Omega_{i}^{0}$, with $\Omega_{i}^{0}=\left\{j \mid n_{i j}=1\right\}$ (note that this definition potentially includes $i$ himself). Then, suppose that agent $i$ 's acquaintance set at $t=1$ consists of the individuals in $\Omega_{i}^{0}$ plus the time-zero acquaintances of the agents in $\Omega_{i}^{0}$. Thus,

$$
\Omega_{i}^{1}=\left\{j \mid j \in \Omega_{i}^{0} \text { or } j \in \Omega_{s}^{0} \text { for } s \in \Omega_{i}^{0}\right\}
$$

Thus, agent $i$ 's acquaintance set at $t=1$ includes the acquaintances of his acquaintances at $t=0$. The acquaintance set expands in each period according to this rule, so that the agent $i$ 's set at time $t+1$ is defined recursively by $\Omega_{i}^{t+1}=\left\{j \mid j \in \Omega_{i}^{t}\right.$ or $j \in \Omega_{s}^{t}$ for $\left.s \in \Omega_{i}^{t}\right\}$.

To represent the evolving acquaintance set in terms of the raw acquaintance matrix, recall the initial discussion of irreducibility in section 2.1. That discussion showed that element $(i, j)$ of the matrix $N^{2}$ equals the number of paths of length 2 between agents $i$ and $j$ via $i$ 's aquaintances. Thus, all individuals $j$ who are acquaintances of $i$ 's time-0 acquaintances are identified by positive values in the $i$ th row of $N^{2}$. As a result, if $N^{2}$ is added to $N$ itself, the $i$ th row of the resulting $N+N^{2}$ matrix has positive elements for agents who are time-0 acquaintances of $i$ or acquaintances of $i$ 's time-0 acquaintances. ${ }^{14}$

Let the matrix $N+N^{2}$, which applies to $t=1$, be denoted $P_{1}$, and consider the matrix $P_{2} \equiv P_{1}+P_{1}^{2}$. By the above reasoning, the $i$ th row of this matrix has positive elements 
for agents who are time-1 acquaintances of $i$ or acquaintances of $i$ 's time-1 acquaintances. Substituting for $P_{1}$ yields $P_{2}=N+2 N^{2}+2 N^{3}+N^{4}$. Generally, by using the recursion rule $P_{t+1}=P_{t}+P_{t}^{2}$, it is easy to see that $P_{t}$ follows the pattern of $P_{2}$, being equal to a weighted sum of all the powers of $N$ up to the power $2 t$. As a result, element $(i, j)$ of $P_{t}$ is equal to a weighted sum of the $(i, j)$ elements of $N^{k}$ for $k=1,2, \cdots 2 t$. But since irreducibility of $N$ means that $N_{i j}^{(k)}>0$ holds for some value of $k$, it follows that this sum must be positive for a sufficiently large value of $t$. This fact in turn implies that all the elements of $P_{t}$ are positive when $t$ is sufficiently large.

Positivity of $P_{t}$ implies a particular form for the row-normalized acquaintance matrix at time $t$. This matrix is denoted $B_{t}$, and it is derived from $P_{t}$ by setting all the positive elements equal to 1 and then row normalizing the resulting matrix. Since all elements of $P_{t}$ are positive when $t$ is large, it follows that, for large $t, B_{t}$ is a matrix with each element equal to $1 / \mathrm{m}$.

To use this fact to make a statement about convergence, let $\widehat{t}$ denote the critical value of $t$ beyond which $P_{t}$ is positive $\widehat{t}$ is the smallest $t^{\prime}$ such that $P_{t}>0$ for $\left.t \geq t^{\prime}\right)$. It is easily seen that $\widehat{t} \leq m$, the dimension of $N .^{15}$ Next, note that the previous law of motion from (3) is rewritten as $\theta^{t+1}=B_{t} \theta^{t}$, with $B_{t}$ taking the place of the constant acquaintance matrix $A$. Then observe that, based on this law and the form of $B_{\hat{t}}, \theta^{\hat{t}+1}=\left(\bar{\theta}^{\hat{t}}, \bar{\theta}^{\hat{t}}, \ldots, \bar{\theta}^{\hat{t}}\right)^{\prime}$, where $\bar{\theta}^{\hat{t}}=\sum_{i=1}^{m} \theta_{i}^{\hat{t}} / m$, the mean attribute value at $t=\widehat{t}$. But since $\theta^{\hat{t}+1}$ has identical elements, it follows that $\theta^{\hat{t}+2}=\theta^{\hat{t}+1}=\left(\bar{\theta}^{\hat{t}}, \bar{\theta}^{\hat{t}}, \ldots, \bar{\theta}^{\hat{t}}\right)^{\prime}$ and that $\theta^{t}$ remains constant at this value for all subsequent $t$ 's. Thus, convergence occurs no later than $\widehat{t}$ (and thus no later than $t=m$ ), yielding

Proposition 5. With an expanding acquaintance set, convergence to a melting-pot equilibrium occurs for any social network where $N$ is irreducible.

The intuition underlying this result is that, when the acquaintance set expands, every agent must eventually become acquainted with everyone else, provided that the initial raw acquaintance matrix $N$ is irreducible. Once this state is reached, the law of motion equates all attributes to a particular mean value, which is then propagated forward.

Numerical examples can illustrate Proposition 5 along with some other features of the 
evolutionary process under an expanding acquaintance set. Accordingly, suppose that the four different $N$ matrices from the examples of section 2.4 are again relevant, but that the acquaintance set expands using the above rules.

The first key feature of the numerical examples is that, under all four cases considered above, convergence to a melting-pot equilibrium occurs. Since the second case considered in section 2.4 was nonconvergent, this difference illustrates Proposition 5, showing that convergence occurs with an expanding acquaintance set even when $A$ is cyclic.

The second notable feature of the examples is that in the third and fourth cases (where one agent was self-referential or two agents had a common acquaintance), convergence is to a different value of $\theta$ than in section 2.4. Attributes converge to melting-pot values of 5.33 and 5.30 in the third and fourth cases, respectively, values that are slightly larger than those reached in the previous examples. However, since attributes in the base case converge to 5.50 in both the basic and expanding-acquaintance models, these limits under the two models may sometimes be same. ${ }^{16}$

Table 5

\section{Attribute Evolution With an Expanding Acquaintance Set}

\begin{tabular}{rrrrrrrrrrr}
\hline \hline$t$ & $\theta_{1}$ & $\theta_{2}$ & $\theta_{3}$ & $\theta_{4}$ & $\theta_{5}$ & $\theta_{6}$ & $\theta_{7}$ & $\theta_{8}$ & $\theta_{9}$ & $\theta_{10}$ \\
\hline 0 & 1.00 & 2.00 & 3.00 & 4.00 & 5.00 & 6.00 & 7.00 & 8.00 & 9.00 & 10.00 \\
1 & 4.33 & 2.00 & 3.00 & 4.00 & 5.00 & 6.00 & 7.00 & 8.00 & 9.00 & 6.66 \\
2 & 5.00 & 4.00 & 3.66 & 4.00 & 5.00 & 6.00 & 7.00 & 7.33 & 7.00 & 6.00 \\
3 & 5.44 & 5.33 & 5.29 & 5.33 & 5.44 & 5.55 & 5.66 & 5.70 & 5.66 & 5.55 \\
4 & 5.50 & 5.50 & 5.50 & 5.50 & 5.50 & 5.50 & 5.50 & 5.50 & 5.50 & 5.50 \\
\hline
\end{tabular}

Consistent with the above discussion, the examples show finally that expansion of the acquaintance set leads to much more rapid convergence to a melting-pot equilibrium. While convergence required 100 periods in the base case of section 2.4, convergence to the meltingpot equilibrium now occurs by $t=4$, as illustrated in Table 5. This rapid convergence, which 
illustrates the fact that a melting-pot equilibrium is reached no later than $t=m=10$, also characterizes the three other cases, all of which converge in four periods.

\subsection{Endogenous linkage intensity}

Suppose that acquaintance sets are again fixed but that each agent is endowed with a fixed total amount of expendable effort that can be used to interact with acquaintances. Let $h_{i j}^{t}$ denote the effort spent by agent $i$ interacting with $j$ at time $t$, with $\sum_{j=1}^{m} h_{i j}^{t}=1$, indicating that available effort is normalized to unity. Note that $h_{i i}^{t}$ can be interpreted as the effort agent $i$ spends in solitary activities.

Suppose that the benefit from an acquaintance depends on the attributes of the other agent as well as the effort devoted to the linkage. Let the time- $t$ benefit that agent $i$ derives from an acquaintance with agent $j$ be written $f\left(h_{i j}^{t}\right) g_{i}\left(\theta_{j}^{t}\right)$, where $f$ is increasing and concave (indicating decreasing returns to effort) and $g_{i}$ is a function whose form may depend on the identity of the agent. Agent $i$ allocates his effort to maximize $\sum_{j=1}^{m} n_{i j} f\left(h_{i j}^{t}\right) g_{i}\left(\theta_{j}^{t}\right)$ subject to $\sum_{j=1}^{m} h_{i j}^{t}=1$. Note that the presence of $n_{i j}$ in the objective function restricts attention to those agents $j$ who are $i$ 's acquaintances.

To consider a simple case, suppose that $f\left(h_{i j}^{t}\right)=\ln \left(h_{i j}^{t}\right)$, and let $g_{i}\left(\theta_{j}^{t}\right)=\theta_{j}^{t} \cdot 17$ The latter assumption says that interaction with higher- $\theta$ agents is more desirable, regardless of the identity of agent $i$. In this case, it is easily seen that the first-order conditions for the maximization problem yield $h_{i j}^{t}=n_{i j} \theta_{j}^{t} /\left(\sum_{l=1}^{m} n_{i l} \theta_{l}^{t}\right)$. This equation says that $h_{i j}^{t}$ equals the share of $\theta_{j}^{t}$ in the aggregate $\theta$ value among $i$ 's acquaintances.

Finally, let the law of motion in (2) be amended so that the influence of acquaintances' attributes depends on the fraction of effort devoted to them:

$$
\theta_{i}^{t+1}=\sum_{j=1}^{m} h_{i j}^{t} \theta_{j}^{t}=\sum_{j=1}^{m}\left(\frac{n_{i j} \theta_{j}^{t}}{\sum_{l=1}^{m} n_{i l} \theta_{l}^{t}}\right) \theta_{j}^{t} .
$$

Note that if all attributes are identical, then (17) reduces to (2).

The terms in parthenses in (18) multiplying the $\theta_{j}^{t}$ 's are not constants, as in (2), but instead depend on the current attribute values in the population. As a result, under this extension of the model, it is not possible to write a simple matrix representation of the law of motion, as 
in (3). Consequently, the theory of irreducible matrices cannot be used to analyze converge, preventing the derivation of any general results.

Numerical examples can be generated, however, and they yield natural conclusions. Using the base-case assumptions from the previous example, it can be shown that population attributes again converge to a melting-pot equilibrium, but the equilibrium attribute value now equals 7.04, which is higher than the previous initial-mean value of 5.50. This increase in equilibrium attributes makes sense given that interaction with higher- $\theta$ agents is more intense under the modified model. The qualitative properties of the other cases considered above are unchanged, with cycling occurring in the second case and melting-pot convergence occurring in the third and fourth cases.

Instead of assuming that agents like interacting with high- $\theta$ individuals, suppose instead that agent $i$ 's time-t benefit from interacting with $j$ is decreasing in the absolute difference between $\theta_{i}^{t}$ and $\theta_{j}^{t}$. Thus, agents prefer to interact with individuals who are like themselves, getting the most benefit in the self-referential case from spending time alone. To operationalize this assumption, let $g_{i}\left(\theta_{j}^{t}\right)=1 /\left[\left(\theta_{j}^{t}-\theta_{i}^{t}\right)^{2}+1\right]$. After making the appropriate modifications to (18), calculations show that convergence to the previous melting-pot value of 5.50 occurs in the base case, a consequence of $g_{i}$ 's symmetry around $\theta_{i}^{t}$. However, because the individuals in this case (who are self-referential) now spend more effort in solitary activities than in interacting with their acquaintances, the convergence process is slowed. Convergence is now achieved at $t=122$ rather than at $t=100$.

Suppose instead that agents are happiest interacting with individuals most different from themselves. To capture this alternative, let $g_{i}\left(\theta_{j}^{t}\right)$ be written as $\left[\left(\theta_{j}^{t}-\theta_{i}^{t}\right)^{2}+1\right]$. Since agents now interact more intensively with dissimilar individuals, the convergence of population attributes is speeded up, with the melting-pot value of 5.50 achieved at $t=76$.

\section{Conclusion}

This paper has linked the two nascent economic literatures on social networks and cultural assimilation by investigating the evolution of population attributes in a simple model where agents are influenced by their acquaintances. The main conclusion of the analysis is that 
attributes converge to a melting-pot equilibrium, where everyone is identical, provided the social network exhibits a sufficient degree of interconnectedness. In one case where convergence does not occur, the evolution of attributes is governed by pure mimickry, with the next-period value of each agent's attributes independent of the current value, being influenced only by the current attributes of acquaintances. In this setting, each agent is, in effect, "not connected to himself," violating one of the sufficient conditions on network interconnectedness.

When the model is extended to allow an expanding acquaintance set, convergence is guaranteed provided a weak interconnectedness condition is satisfied (irreducibility), and convergence is rapid. If the intensity of interactions with acquaintances becomes endogenous, convergence (when it occurs) is slowed when agents prefer to interact with people like themselves and hastened when interaction with dissimilar agents is preferred.

The model analyzed in the paper is exceedingly simple, and except for the second extension, it does not permit agents to make any economic decisions. Future work could attempt enrich the framework by incorporating such decisions. One natural modification would allow the social network to be endogenous by including linkage decisions on the part of agents. Given the intense current interest in social networks among economists, the payoff to future work on models like the present one is likely to be high. 


\section{References}

Alesina, A., LA Ferrara, E., 2004. Ethnic diversity and economic performance. National Bureau of Economic Research Working Paper \#10313.

Bala, V., Goyal, S., 2000. A noncooperative model of network formation. Econometrica $68,1181-1229$.

Ballester, C., Calvó-Armengol, A., Zenou, Y., 2004. Who's who in crime networks: Wanted the key player. Unpublished paper, Universitat Autònoma de Barcelona.

Bisin, A., Verdier, T., 2001. The economics of cultural transmission and the dynamics of preferences. Journal of Economic Theory 97, 298-319.

Bisin, A., Verdier, T., 2000. Beyond the melting pot: Cultural transmission, marriage, and the evolution of ethnic and religious traits. Quarterly Journal of Economics 115, 955-988.

BrUECKNER, J.K., 2004. Friendship networks, Unpublished paper, University of Illinois at Urbana-Champaign.

Goyal, S., 2003. Learning in networks. In: Demange, G. and Wooders, M. (Eds.), Group Formation in Economics: Networks, Clubs, and Coalitions. Cambridge University Press, Cambridge, forthcoming.

Granovetter, M., 1973. The strength of weak ties. American Journal of Sociology 78, 1360-1380.

Jackson, M.O., Wolinsky, A., 1996. A strategic model of social and economic networks. Journal of Economic Theory 71, 44-74.

JACKSOn, M.O., 2004. A survey of models of network formation: Stability and efficiency. In: Demange, G. and Wooders, M. (Eds.), Group Formation in Economics: Networks, Clubs, and Coalitions. Cambridge University Press, Cambridge, forthcoming.

KÓNyA, I., 2002. A dynamic model of cultural assimilation. Unpublished paper, Boston College.

Seneta, E., 1973. Non-Negative Matrices: An Introduction to Theory and Applications. George Allen \& Unwin, London.

Strang G., 1976. Linear Algebra and its Applications. Academic Press, New York. 
McPherson, M., Smith-Lovin, L., Cook, J.M., 2001. Birds of a feather: Homophily in social networks. Annual Review of Sociology 27, 415-444.

Wasserman, S., Faust, K., 1994. Social Network Analysis: Methods and Applications. Cambridge, Cambridge University Press. 


\section{Footnotes}

*We thank Kangoh Lee for helpful comments. Any shortcomings in the paper, however, are our responsibility.

${ }^{1}$ For examples of the sociological research on social networks, see Granovetter (1973) and Wasserman and Faust (1994).

${ }^{2}$ In a related paper, Bisin and Verdier (2000) study the effect of marriage choices on cultural assimilation.

${ }^{3}$ This behavior is known "homophily" in the sociology literature (see McPherson, Smith-Lovin and Cook (2001)).

${ }^{4}$ More generally, suppose that both agents $i$ and $j$ are acquainted with agent $s$. Then, the $i$ th and $j$ th rows of $N$ have a 1 in the $s$ th spot, and as a result, the inner product of the $i$ th row and the $j$ th column of $N$ (which equals the $j$ th row) contains at least one unitary term, which counts the length-2 path between $i$ and $j$ via $s$. If $i$ and $j$ are both acquainted with a second individual $k$ (but have no additional common acquaintances), then the given inner product has two unitary terms, indicating the existence of 2 length-2 paths between $i$ and $j$. The result is a value of 2 for element $(i, j)$ of $N^{2}$. Continuing the argument, if agent $j$ is acquainted with another agent $l$, then there exist at least two length-3 paths between $i$ and $l$ (via $s$ and $j$ and via $k$ and $j$ ). This fact is reflected in the inner product of the $i$ th row of $N^{2}$ and the $l$ th column of $N$, which has a 1 in its $j$ th spot. This inner product, which equals element $(i, l)$ of $N^{3}$, is at least 2 , indicating the existence of at least 2 paths of length 3 between $i$ and $l$.

${ }^{5}$ This discussion follows Strang (1976, ch. 5).

${ }^{6}$ The equality $S^{-1}=S^{\prime}$ follows from the fact that the eigenvectors of a symmetric matrix are orthogonal, which in turn yields $S^{\prime} S=I$, where $I$ is the identity matrix (the eigenvectors are normalized to have unit length). To establish orthogonality, premultiply both sides of the equation $A S_{i}=\lambda_{i} S_{i}$ by $S_{j}^{\prime}$ to yield $S_{j}^{\prime} A S_{i}=\lambda_{i} S_{j}^{\prime} S_{i}$. Then transpose both sides of the equation $A S_{j}=\lambda_{j} S_{j}$ and postmultiply by $S_{i}$ to yield $S_{j}^{\prime} A^{\prime} S_{i}=\lambda_{j} S_{j}^{\prime} S_{i}$. When $A$ is symmetric, the LHS terms of the second and fourth equations are equal, implying equality of the RHS terms. But since eigenvalues are non-zero and $\lambda_{i} \neq \lambda_{j}$, this equality requires $S_{i}^{\prime} S_{j}=0$.

${ }^{7}$ The constancy of the mean attribute level can be demonstrated by recalling that eigenvectors 
associated with $A$ 's unit eigenvalue are proportional to the unit vector. Thus, consider the vector $\widetilde{S}_{1}=\left(\frac{1}{m}, \frac{1}{m} \cdots \frac{1}{m}\right)^{\prime}$, which satisfies $A \widetilde{S}_{1}=\widetilde{S}_{1}$ and hence $\widetilde{S}_{1}^{\prime} A=\widetilde{S}_{1}^{\prime}$. As a result, $\widetilde{S}_{1}^{\prime} A \theta^{t}=\widetilde{S}_{1}^{\prime} \theta^{t}=\bar{\theta}^{t}$, where $\bar{\theta}^{t}$ is the mean attribute value at time $t$. But, using the law of motion $\theta^{t+1}=A \theta^{t}$, it then follows that $\bar{\theta}^{t+1}=\widetilde{S}_{1}^{\prime} \theta^{t+1}=\widetilde{S}_{1}^{\prime} A \theta^{t}=\bar{\theta}^{t}$. Thus, the mean attribute level is constant over time and equal to $\bar{\theta}^{0}$ for all $t$.

${ }^{8}$ See Seneta (1973, ch. 1) for a discussion of the period concept and related results.

${ }^{9}$ At $t=100$, equality of attributes holds to the 5 significant digits generated by the computer program leading to Table 1.

${ }^{10}$ The absence of self-referential agents does not always lead to cyclic behavior. For example, it can be shown that if the number of agents is odd rather than even, convergence to $\bar{\theta}^{0}$ occurs.

${ }^{11}$ Convergence at the 5 -significant digit level, however, does not occur until $t$ passes 300.

${ }^{12}$ Convergence at the 5-significant digit level does not occur until $t$ passes 190.

${ }^{13}$ In another interesting case, the social network contains a "key player," who is acquainted with many other agents, as in Ballester, Calvó-Armengol and Zenou (2004) (Brueckner (2004) considers a similar case). To investigate the key-player case, let the $N$ matrix in (16) be modified by assuming that one individual $k$ is acquainted with all the other agents, while the other agents only have individual $k$ as an acquaintance. With all agents assumed to be self-referential, the $k$ th row and column of $N$ are then vectors of 1's, with the remaining diagonal elements also equal to 1 . Numerical examples using such a matrix expose two regularities. First, convergence to a melting-pot equilibrium is rapid, occurring in about 20 periods. Second, the melting-pot attribute value lies between the mean initial attribute level and the key player's attribute value. Note that fast convergence means that the non-unitary eigenvalues of $N$ in the key-player case are small in absolute value.

${ }^{14}$ Note that some agents may belong to both groups.

${ }^{15}$ The reason is that any non-repetitive path between two agents (or between an agent and himself) can never be longer than $m$, which implies that all agents must be connected once $m$ periods have elapsed.

${ }^{16}$ Although attributes converge to the mean initial value in the basic model when the number of initial acquaintances is the same across agents, this outcome may not be a general property 
of the expanding-acquaintance model despite the evidence of the numerical example. If it were possible to establish that $B_{t}$ is symmetric for all $t$, then the argument of footnote 7 could be used to show that the mean attributes are constant over time. This conclusion in turn would imply that the common attribute value reached in the expanding-acquaintance melting-pot equilibrium equals the initial mean value. However, even though it is possible to establish that $P_{t}$ for $t \geq 1$ is symmetric and has a constant row sum, it does not appear possible to establish the constancy of the row sum of the (symmetric) incidence matrix of $P_{t}$ (thus, the number of acquaintances may differ across agents for $t \geq 1$ ). As a result, each row of this incidence matrix need not be divided by the same number in generating $B_{t}$, which means that the latter matrix may not be symmetric.

${ }^{17}$ Note that since $h_{i j}^{t}<1$, the objective function is negative given use of natural log function. This is a scaling issue, however, that can be ignored. 


\section{CESifo Working Paper Series}

(for full list see www.cesifo.de)

1257 François Larmande and Jean-Pierre Ponssard, EVA and the Controllability-congruence Trade-off: An Empirical Investigation, August 2004

1258 Vesa Kanniainen and Jenni Pääkkönen, Anonymous Money, Moral Sentiments and Welfare, August 2004

1259 Panu Poutvaara and Andreas Wagener, Why is the Public Sector More Labor-Intensive? A Distortionary Tax Argument, August 2004

1260 Lars P. Feld and Stefan Voigt, Making Judges Independent - Some Proposals Regarding the Judiciary, August 2004

1261 Joop Hartog, Hans van Ophem, and Simona Maria Bajdechi, How Risky is Investment in Human Capital?, August 2004

1262 Thomas Eichner and Rüdiger Pethig, Efficient Nonanthropocentric Nature Protection, August 2004

1263 David-Jan Jansen and Jakob de Haan, Look Who's Talking: ECB Communication during the First Years of EMU, August 2004

1264 David F. Bradford, The X Tax in the World Economy, August 2004

1265 Hans-Werner Sinn, Migration, Social Standards and Replacement Incomes. How to Protect Low-income Workers in the Industrialized Countries against the Forces of Globalization and Market Integration, August 2004

1266 Wolfgang Leininger, Fending off one Means Fending off all: Evolutionary Stability in Submodular Games, August 2004

1267 Antoine Bommier and Bertrand Villeneuve, Risk Aversion and the Value of Risk to Life, September 2004

1268 Harrie A. A. Verbon and Lex Meijdam, Too Many Migrants, Too Few Services: A Model of Decision-making on Immigration and Integration with Cultural Distance, September 2004

1269 Thomas Eichner and Rüdiger Pethig, Economic Land Use, Ecosystem Services and Microfounded Species Dynamics, September 2004

1270 Federico Revelli, Performance Rating and Yardstick Competition in Social Service Provision, September 2004

1271 Gerhard O. Orosel and Klaus G. Zauner, Vertical Product Differentiation When Quality is Unobservable to Buyers, September 2004 
1272 Christoph Böhringer, Stefan Boeters, and Michael Feil, Taxation and Unemployment: An Applied General Equilibrium Approach, September 2004

1273 Assaf Razin and Efraim Sadka, Welfare Migration: Is the Net Fiscal Burden a Good Measure of its Economics Impact on the Welfare of the Native-Born Population?, September 2004

1274 Tomer Blumkin and Volker Grossmann, Ideological Polarization, Sticky Information, and Policy Reforms, September 2004

1275 Katherine Baicker and Nora Gordon, The Effect of Mandated State Education Spending on Total Local Resources, September 2004

1276 Gabriel J. Felbermayr and Wilhelm Kohler, Exploring the Intensive and Extensive Margins of World Trade, September 2004

1277 John Burbidge, Katherine Cuff and John Leach, Capital Tax Competition with Heterogeneous Firms and Agglomeration Effects, September 2004

1278 Joern-Steffen Pischke, Labor Market Institutions, Wages and Investment, September 2004

1279 Josef Falkinger and Volker Grossmann, Institutions and Development: The Interaction between Trade Regime and Political System, September 2004

1280 Paolo Surico, Inflation Targeting and Nonlinear Policy Rules: The Case of Asymmetric Preferences, September 2004

1281 Ayal Kimhi, Growth, Inequality and Labor Markets in LDCs: A Survey, September 2004

1282 Robert Dur and Amihai Glazer, Optimal Incentive Contracts for a Worker who Envies his Boss, September 2004

1283 Klaus Abberger, Nonparametric Regression and the Detection of Turning Points in the Ifo Business Climate, September 2004

1284 Werner Güth and Rupert Sausgruber, Tax Morale and Optimal Taxation, September 2004

1285 Luis H. R. Alvarez and Erkki Koskela, Does Risk Aversion Accelerate Optimal Forest Rotation under Uncertainty?, September 2004

1286 Giorgio Brunello and Maria De Paola, Market Failures and the Under-Provision of Training, September 2004

1287 Sanjeev Goyal, Marco van der Leij and José Luis Moraga-González, Economics: An Emerging Small World?, September 2004 
1288 Sandro Maffei, Nikolai Raabe and Heinrich W. Ursprung, Political Repression and Child Labor: Theory and Empirical Evidence, September 2004

1289 Georg Götz and Klaus Gugler, Market Concentration and Product Variety under Spatial Competition: Evidence from Retail Gasoline, September 2004

1290 Jonathan Temple and Ludger Wößmann, Dualism and Cross-Country Growth Regressions, September 2004

1291 Ravi Kanbur, Jukka Pirttilä and Matti Tuomala, Non-Welfarist Optimal Taxation and Behavioral Public Economics, October 2004

1292 Maarten C. W. Janssen, José Luis Moraga-González and Matthijs R. Wildenbeest, Consumer Search and Oligopolistic Pricing: An Empirical Investigation, October 2004

1293 Kira Börner and Christa Hainz, The Political Economy of Corruption and the Role of Financial Institutions, October 2004

1294 Christoph A. Schaltegger and Lars P. Feld, Do Large Cabinets Favor Large Governments? Evidence from Swiss Sub-Federal Jurisdictions, October 2004

1295 Marc-Andreas Mündler, The Existence of Informationally Efficient Markets When Individuals Are Rational, October 2004

1296 Hendrik Jürges, Wolfram F. Richter and Kerstin Schneider, Teacher Quality and Incentives: Theoretical and Empirical Effects of Standards on Teacher Quality, October 2004

1297 David S. Evans and Michael Salinger, An Empirical Analysis of Bundling and Tying: Over-the-Counter Pain Relief and Cold Medicines, October 2004

1298 Gershon Ben-Shakhar, Gary Bornstein, Astrid Hopfensitz and Frans van Winden, Reciprocity and Emotions: Arousal, Self-Reports, and Expectations, October 2004

1299 B. Zorina Khan and Kenneth L. Sokoloff, Institutions and Technological Innovation During Early Economic Growth: Evidence from the Great Inventors of the United States, 1790 - 1930, October 2004

1300 Piero Gottardi and Roberto Serrano, Market Power and Information Revelation in Dynamic Trading, October 2004

1301 Alan V. Deardorff, Who Makes the Rules of Globalization?, October 2004

1302 Sheilagh Ogilvie, The Use and Abuse of Trust: Social Capital and its Deployment by Early Modern Guilds, October 2004

1303 Mario Jametti and Thomas von Ungern-Sternberg, Disaster Insurance or a Disastrous Insurance - Natural Disaster Insurance in France, October 2004 
1304 Pieter A. Gautier and José Luis Moraga-González, Strategic Wage Setting and Coordination Frictions with Multiple Applications, October 2004

1305 Julia Darby, Anton Muscatelli and Graeme Roy, Fiscal Federalism, Fiscal Consolidations and Cuts in Central Government Grants: Evidence from an Event Study, October 2004

1306 Michael Waldman, Antitrust Perspectives for Durable-Goods Markets, October 2004

1307 Josef Honerkamp, Stefan Moog and Bernd Raffelhüschen, Earlier or Later: A General Equilibrium Analysis of Bringing Forward an Already Announced Tax Reform, October 2004

1308 M. Hashem Pesaran, A Pair-Wise Approach to Testing for Output and Growth Convergence, October 2004

1309 John Bishop and Ferran Mane, Educational Reform and Disadvantaged Students: Are They Better Off or Worse Off?, October 2004

1310 Alfredo Schclarek, Consumption and Keynesian Fiscal Policy, October 2004

1311 Wolfram F. Richter, Efficiency Effects of Tax Deductions for Work-Related Expenses, October 2004

1312 Franco Mariuzzo, Patrick Paul Walsh and Ciara Whelan, EU Merger Control in Differentiated Product Industries, October 2004

1313 Kurt Schmidheiny, Income Segregation and Local Progressive Taxation: Empirical Evidence from Switzerland, October 2004

1314 David S. Evans, Andrei Hagiu and Richard Schmalensee, A Survey of the Economic Role of Software Platforms in Computer-Based Industries, October 2004

1315 Frank Riedel and Elmar Wolfstetter, Immediate Demand Reduction in Simultaneous Ascending Bid Auctions, October 2004

1316 Patricia Crifo and Jean-Louis Rullière, Incentives and Anonymity Principle: Crowding Out Toward Users, October 2004

1317 Attila Ambrus and Rossella Argenziano, Network Markets and Consumers Coordination, October 2004

1318 Margarita Katsimi and Thomas Moutos, Monopoly, Inequality and Redistribution Via the Public Provision of Private Goods, October 2004

1319 Jens Josephson and Karl Wärneryd, Long-Run Selection and the Work Ethic, October 2004

1320 Jan K. Brueckner and Oleg Smirnov, Workings of the Melting Pot: Social Networks and the Evolution of Population Attributes, October 2004 